\title{
Gold Films in Solar Energy Utilisation
}

\section{B. J. Brinkworth}

Department of Mechanical Engineering, University College, Cardiff, Wales

\section{Examples are given of some of the many possible uses of gold films in devices for converting solar energy. There could be a significant role for gold in this rapidly expanding field.}

The disturbances that have led to a greatlyincreased price of oil have accelerated the studies of alternative sources of energy which had already been proceeding steadily for some time. Among these are developments leading to the wider utilisation of solar energy. It is noteworthy, for example, that funding of solar energy research by the U.S. National Science Foundation has jumped from $\$ 1.6$ million for $1971-72$ to $\$ 14.1$ million for $1973-74$. Though other countries are unlikely to match this massive investment, the pace of solar energy research has sharply increased in many parts of the world.

The characteristics of all solar energy devices are dependent upon the optical properties of the receiving surfaces. These properties are amenable to modification to some extent by surface treatments, among which are electroplating and other methods for the deposition of metals. It is likely that the optical properties of gold films, combined with the unusual chemical inertness of the metal, will be strong recommendations for their use in this rapidly developing field.

\section{Solar Heaters}

Solar heaters for domestic hot water or space heating already have a respectable history. Millions are now in use in several countries and the number is certain to increase greatly in coming years. The most elementary type of heater is illustrated in Figure 1. Solar radiation passes through the transparent cover plate and is absorbed on the collector plate behind it. This is in contact with the heat-transfer fluid which is then warmed and passed to a storage tank by natural convection or through the action of a circulating pump. The efficiency of conversion of solar energy into heat in the fluid depends upon the temperature at which the collector is allowed to operate. As the collector plate heats up, it emits radiation which is intercepted by the cover plate. The cover plate becomes warmed in turn and eventually passes some of the energy into the surroundings by convection and radiation.

Evidently it is desirable to keep these losses to the surroundings to a minimum. The action of the cover plate contributes greatly to this, in the manner of the glass of a greenhouse. The solar radiation has a distribution with wavelength approximating to that of a black-body radiator at a temperature of about $6000 \mathrm{~K}$. As seen from Figure 2, the peak of the energy density comes at about $500 \mathrm{~nm}$ at this temperature and only a little of the energy occurs at wavelengths greater than $2000 \mathrm{~nm}$. Most glasses transmit radiation well at these wavelengths; typically about 85 per cent of the sun's radiation will be passed.

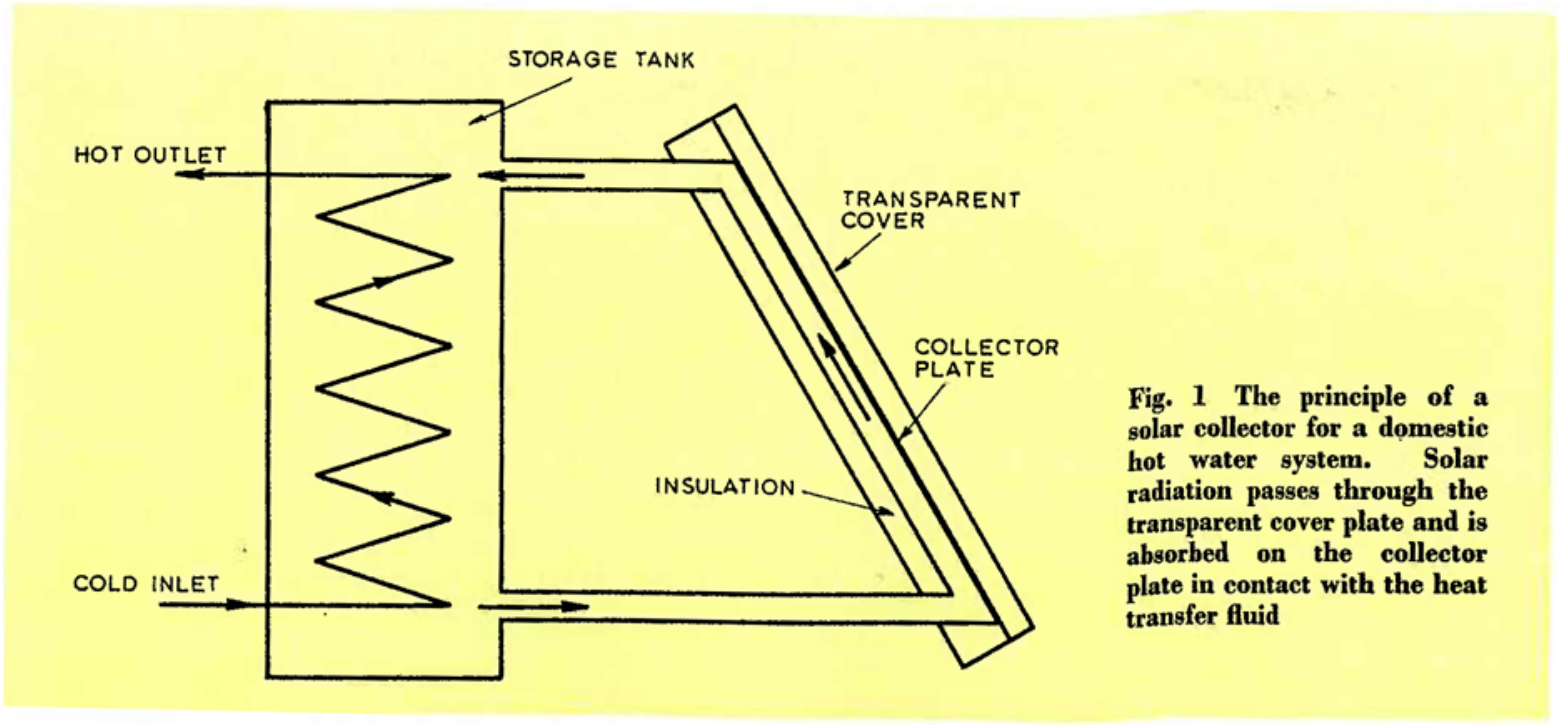




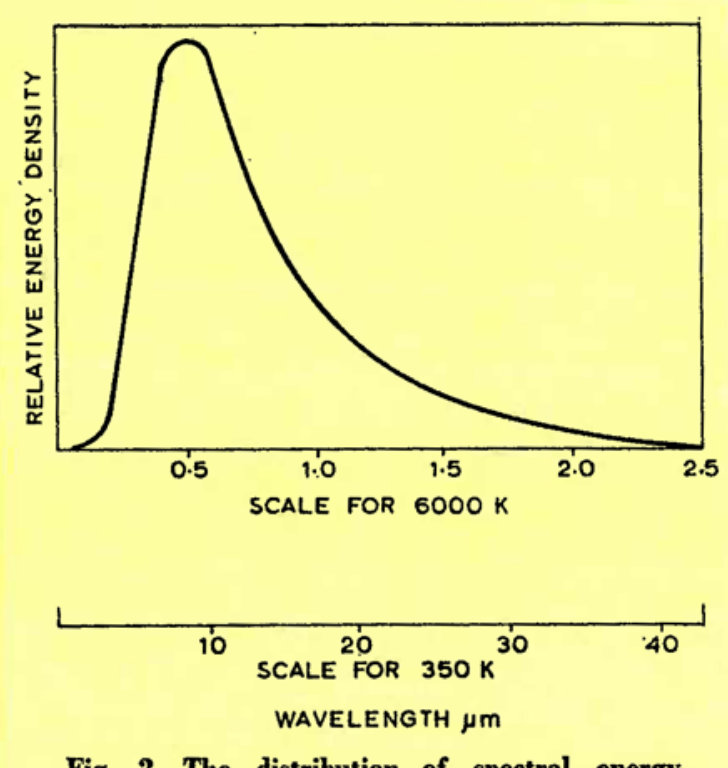

Fig. 2 The distribution of spectral energy at various temperatures

The radiation from the collector plate, on the other hand, will have a distribution like that of a radiator at the operating temperature, which will probably not be more than about $350 \mathrm{~K}\left(77^{\circ} \mathrm{C}\right)$. The peak energy density at this temperature comes, as shown, at a wavelength of about $8000 \mathrm{~nm}$, where glass is quite opaque.

The radiation exchange between the collector and the cover plate can be minimised by treatments affecting their emissivities at operating temperatures, say $\varepsilon_{1}$ and $\varepsilon_{2}$ respectively. The nett radiation exchange is proportional to the function $\varepsilon$ given by:

$$
\frac{\varepsilon}{1}=\frac{1}{\varepsilon_{1}}+\frac{1}{\varepsilon_{2}}-1
$$

It is evident that $\varepsilon_{1}$ and $\varepsilon_{2}$ should be kept as small as possible. If they both have the value 1 of black bodies, the effective emissivity $\varepsilon$ will also be 1 . If they can be reduced to 0.1 , then $\varepsilon$ becomes 0.053 ; if only one is reduced to $0.1, \varepsilon$ becomes 0.1 .

Low values of emissivity at moderate temperatures are characteristics of bulk metals if they have a good surface finish. A thick gold film on the collector plate, for example, would give it an emissivity of less than 0.05 (1). However, the absorptivity at the shorter wave-lengths of the solar radiation would be only about 0.4 (2), representing a needless waste of the incident

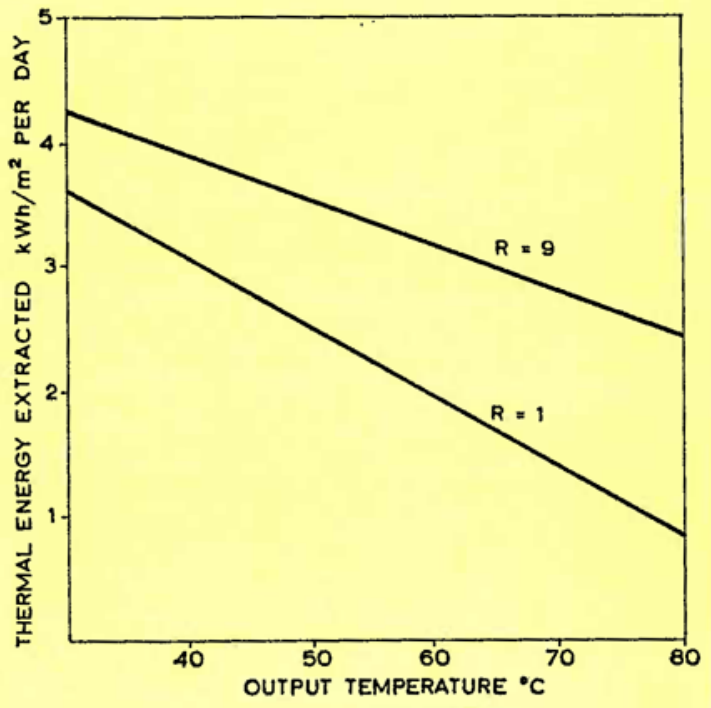

Fig. 3 The output of thermal energy from a flatplate collector in a water heater operating in a latitude of $23 \frac{1}{2}^{\circ}$. Treatment of the collector plate to give a high ratio $R$ of solar absorptivity to long-wave emissivity increases the output at a given temperature

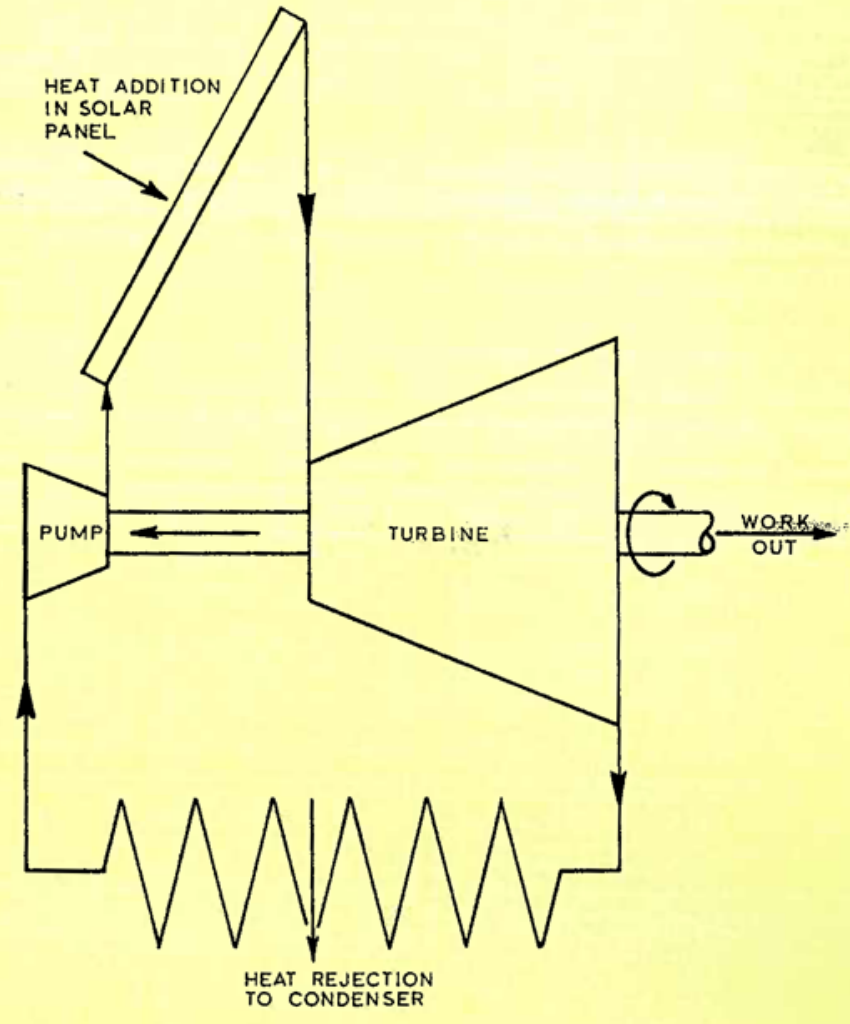

Fig. 4 The principle of an elementary solar-powered heat engine 
Fig. 5 The efficiency of a solar-powered heat engine showing the marked effect of treating the collector plate to give a high ratio of solar absorptivity to emissivity

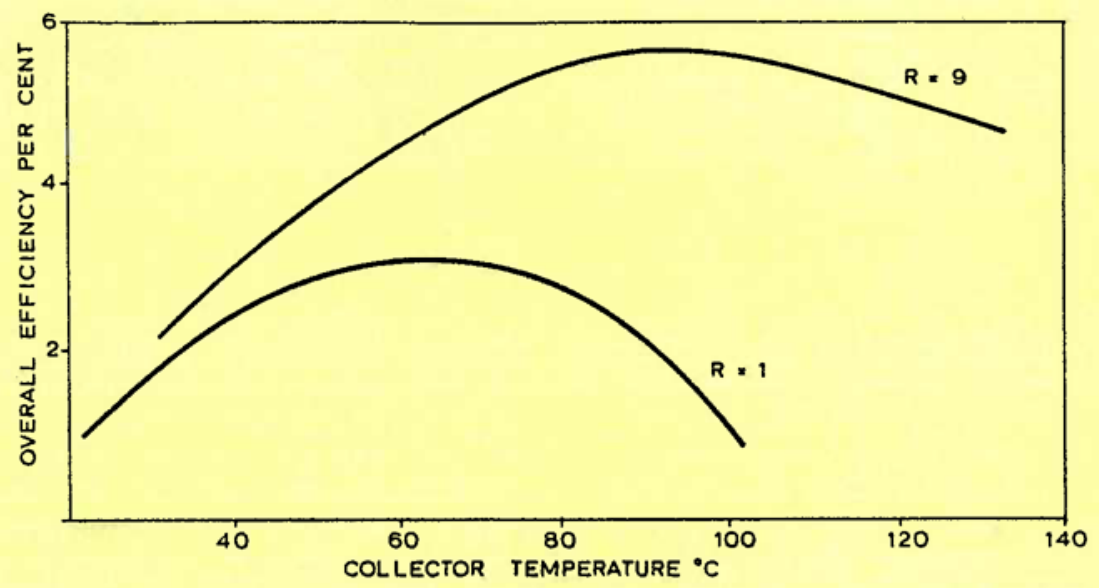

znergy. If a film only a few nm thick were employed, this would retain the low emissivity at long wavelengths, while becoming virtually transparent at solar wavelengths. Then the absorptivity of the substrate material at these wavelengths determines the overall absorptivity of the plate. This absorptivity may be made to approach the value of 1 .

In this way, it will be seen that a ratio $R$ of solar absorptivity to long-wavelength emissivity of 10 or more is accessible, with an acceptably high value of absorptivity. A similar result may be obtained by utilising the properties of the opposite kind exhibited by a layer of gold particles laid down from a smoke (3). When the particles are of an appro- priate size, they absorb strongly at the short wavelengths of the solar spectrum, but do not impede long-wavelength radiation emitted from the substrate. In this case, the substrate needs a low emissivity and this could also be provided by a gold film.

The effect of such treatments on the performance of the collector is quite marked. Figure 3, for example, shows the energy extractable from a certain type of water heater (4) when the collector surface is treated to give $R=1$ and $R=9$. It is noteworthy that in this example the energy recovery is increased by a factor of two at $70^{\circ} \mathrm{C}$.

The transparency of thin gold films without a corresponding loss of low-temperature emissivity may
Fig. 6 The efficiency of a system can be raised by concentrating the solar energy from a large area on to a smaller one by reflection. Two such schemes are shown here; gold films might be employed on both the reflecting and the absorbing surfaces

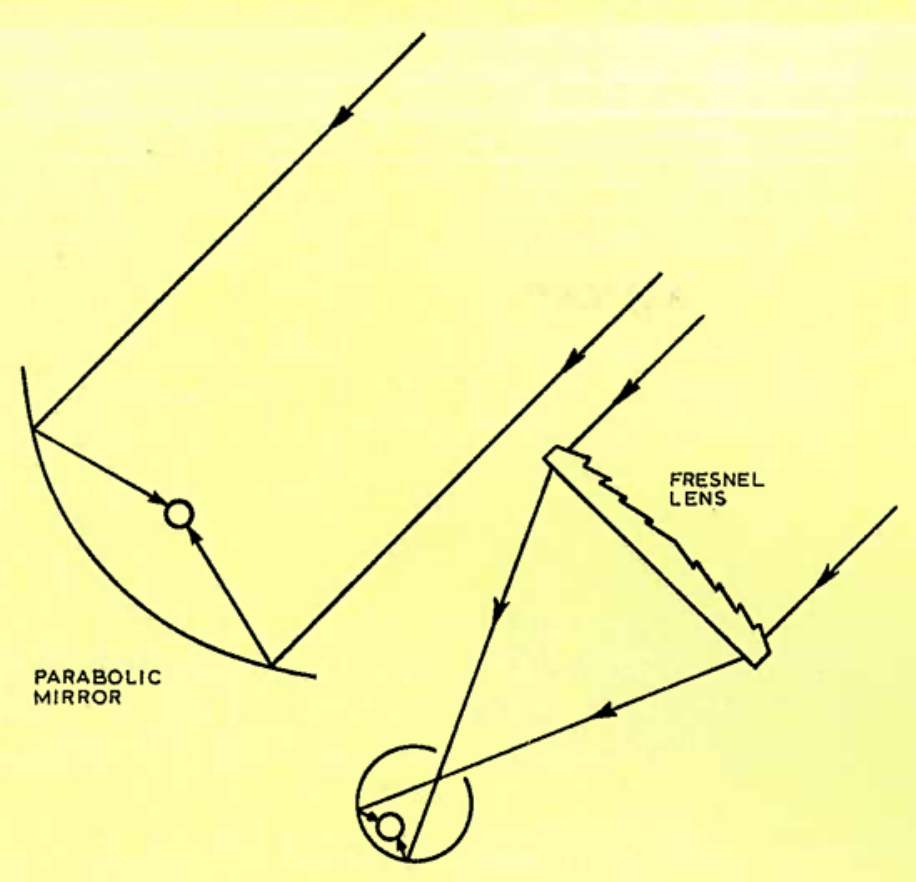


make them attractive also for the treatment of cover plates, bringing yet further benefits to the overall performance.

\section{Solar-powered Engines}

If the collector is employed as the heat source for a thermodynamic engine, say of the type shown in Figure 4, the effect of the surface treatment on the performance is equally marked (4). This is because the efficiency of a thermodynamic device is a function of the difference between the maximum and minimum temperatures of the cycle. As seen from Figure 3, the energy available from the collector falls as the output temperature rises, but the efficiency of the engine increases, so that the overall efficiency passes through a maximum at some temperature. This is illustrated in Figure 5, which shows clearly the strong effect of collector characteristics on the system efficiency (4).

The efficiency of a low-temperature system such as that of Figure 4 may be rather low to be of widespread value. It can be raised only by operating at higher temperatures and to obtain these the solar energy falling on a large area must be concentrated on to a smaller one. Two such schemes are illustrated in Figure 6. It will be seen that both involve reflection of solar radiation, as well as absorption with a selective coating as for the flat collector. In the more elaborate scheme (5) one reflective coating has to be applied on glass. Though gold films do not have a very high reflectivity at solar wavelengths, this can be obtained with some of its alloys $(1,2)$. It is possible that the great chemical stability of gold and gold alloys may give them a competitive advantage over other materials for long-term use in situations like these.

The fact that the desired selective optical properties may be obtained with films of such small thickness means that it becomes possible to consider using an expensive metal at quite modest cost. A place for gold films in solar energy utilisation seems assured.

\section{Acknowledgement}

Some of the illustrations for this paper are taken from the author's book (4) by agreement with the publisher, Compton-Russell Ltd, Salisbury, England.

\section{References}

1 R. C. Langley, Gold Bulletin, 1971, 4, 62-66

2 O. Loebich, Gold Bulletin, 1972, 5, 2-10

3 L. Harris, R. T. McGinnies and B. M. Siegel, F. Opt. Soc. Am., 1948, 38, 582-589

4 B. J. Brinkworth, Solar Energy for Man, Compton Press, 1972, ISBN 900193131

5 A. B. Meinel and M. P. Meinel, Physics Today, 1972, 25, $44-50$

\section{The Tribological Behaviour of Gold}

\section{A BASIC STUDY OF FRICTION AND WEAR}

The importance attached to the wearing and frictional properties of gold has increased greatly with its extensive applications in the form of sliding contacts in the electronics industry, and a number of papers have been published describing practical investigations in this field. A more fundamental study of the characteristics of a gold-to-gold system under carefully controlled rubbing conditions has recently been reported by Harmut Frey and Heinz-Gerhard Feller working in the Institut für Metallforschung in the Technical University of Berlin (Z. Metallkunde, 1973, 64, (8), 529-536).

The method employed was to cause a small cylinder of polycrystalline gold to be moved across the surface of a single crystal of gold, both being of 99.999 per cent purity. The depth and width of the wear track could be measured; so too could the size and nature of wear products.

It was observed that after an initial "wear-free" period of operation, characterised by a relatively low coefficient of friction which in turn had been shown to vary according to the crystallographic plane, the coefficient of friction between the two surfaces increased rapidly and a wear track appeared on the roller. Simultaneously gold was transferred from the roller to the single crystal and wear particles appeared. As the experiment continued the coefficient of friction decreased, as too did wear, until a condition more or less of equilibrium was established again with a relatively low coefficient of friction and insignificant wear. The "wear-free" period is of special significance. Its duration depends on load and so too does the nature of the wear track, and this must point to the advantages of keeping contact force to a minimum.

Examination of the wear products by scanning electron microscope showed that the form and size of the wear particles depended on test conditions. At light loads most of the metal transferred from the roller was in the form of small flat particles that were directly sheared off the parent surface. In time these broke down into flakes. Particle size also depended on load. In one example, with a load of $10 \mathrm{~g}$ the number of particles was more than four times as great as with $100 \mathrm{~g}$ although in the former case the mean diameter of the particles was barely one third.

The underlying causes of these phenomena are complex. The authors suggest that adhesion is responsible not only for friction but also for material transfer, although loose particles develop as a result of mechanical fatigue of the surfaces. The melting of individual microcontacts also plays a part in the overall phenomena. With this in mind they suggest that in further investigations of this nature attention should be directed to the basic principles of elastic and plastic deformation and fracture.

J. G. W. 